\title{
Article \\ Green Synthesis of Iron Nanoparticles Using Green Tea and Its Removal of Hexavalent Chromium
}

\author{
Runqin Hao ${ }^{1,2}$, Dong $\mathrm{Li}^{1, *}$, Jie Zhang ${ }^{1,3}$ and Tifeng Jiao ${ }^{4, *}$ \\ 1 Key Laboratory of Water Quality Science and Water Environment Recovery Engineering, \\ Beijing University of Technology, Beijing 100124, China; hrunqin@163.com (R.H.); 6282031@163.com (J.Z.) \\ 2 Environmental Protection Research Institute of Light Industry, Beijing 100089, China \\ 3 State Key Laboratory of Urban Water Resource and Environment, Harbin Institute of Technology, \\ Harbin 150090, China \\ 4 State Key Laboratory of Metastable Materials Science and Technology, Yanshan University, \\ Qinhuangdao 066004, China \\ * $\quad$ Correspondence: lidong2006@bjut.edu.cn (D.L.); tfjiao@ysu.edu.cn (T.J.); Tel.: +86-10-67392099-4 (D.L.)
}

Citation: Hao, R.; Li, D.; Zhang, J.; Jiao, T. Green Synthesis of Iron Nanoparticles Using Green Tea and Its Removal of Hexavalent Chromium. Nanomaterials 2021, 11, 650. https://doi.org/10.3390/ nano11030650

Academic Editor: George Z. Kyzas

Received: 6 February 2021

Accepted: 26 February 2021

Published: 8 March 2021

Publisher's Note: MDPI stays neutral with regard to jurisdictional claims in published maps and institutional affiliations.

Copyright: ( $\odot 2021$ by the authors. Licensee MDPI, Basel, Switzerland. This article is an open access article distributed under the terms and conditions of the Creative Commons Attribution (CC BY) license (https:// creativecommons.org/licenses/by/ $4.0 /)$.

\begin{abstract}
Chromium (VI) is a ubiquitous groundwater contaminant and it is dangerous to both ecological and human health. Iron nanoparticles $(n \mathrm{Fe})$ have a large specific surface area and they are highly efficient in removing chromium (VI) from aqueous solution. However, since the traditional reductive synthesis of $n \mathrm{Fe}$ is relatively expensive and often causes secondary pollution, it is necessary to develop a low-cost green synthetic method using plant extracts. Synthetic conditions are important for obtaining highly active chromium-removing nanomaterials. In this paper, a green tea extract was used to prepare $n \mathrm{Fe}$ and the effects of synthetic conditions on subsequent remediation performance were investigated. The optimal conditions included a green tea extract $/ \mathrm{Fe}^{2+}$ ratio of 1:2 (91.6\%), a green tea extract temperature of $353 \mathrm{~K}(88.3 \%)$ and a synthetic temperature of $298 \mathrm{~K}(88.1 \%)$. Advanced material characterization techniques, including XPS, SEM-EDS, TEM, and BrunauerEmmett-Teller (BET) confirmed that the average particle size was between $50-80 \mathrm{~nm}$, with a specific surface area of $42.25 \mathrm{~m}^{2} \cdot \mathrm{g}^{-1}$. Furthermore $n$ Fe had a core-shell structure, where Fe (0) constituted the core and a shell was composed of iron oxide. Finally, a mechanism for synthesizing $n$ Fe by green tea extract was proposed, providing a theoretical basis for optimized synthetic conditions for preparing $n$ Fe when using green tea extract.
\end{abstract}

Keywords: $\mathrm{Cr}(\mathrm{VI})$; iron nanoparticles; green tea extract; removal efficiency

\section{Introduction}

Many common industrial activities, such as electroplating, printing, dying and leather processing generate large volumes of metal-rich waste streams, which without appropriate treatment can lead to significant contamination of surface water, groundwater and soil. Of the large number of metal contaminants deposited into the environment, chromium $(\mathrm{Cr})$ is one metal that has attracted much concern owing to its high toxicity even at very low concentrations [1,2]. While chromium exists in two major oxidation states, $\mathrm{Cr}(\mathrm{III})$ and $\mathrm{Cr}(\mathrm{VI}), \mathrm{Cr}(\mathrm{VI})$ is 500 times more toxic than $\mathrm{Cr}(\mathrm{III})$; it is mainly $\mathrm{Cr}(\mathrm{VI})$ exposure that is associated with human health risks, as $\mathrm{Cr}(\mathrm{VI})$ toxicity may damage human organs including the kidneys and liver, cause dermatitis and trigger gastrointestinal ulcers [1,2]. Therefore, removing chromium from wastewater is urgent. Traditional methods including adsorption, chemical precipitation, electrocoagulation, ion exchange, electrodialysis, and membrane separation are usually used to remove chromium. Adsorption has a high removal efficiency, simple operation and low cost, so it has emerged as the best way to remove chromium from wastewater. Recently, since reducing the levels of toxic substances in aquatic ecosystems and promoting water reuse after treatment has become more popular, calls to use nanomaterials for environmental remediation have gained much momentum [3-5]. Until 
now, various materials have been successfully used for $\mathrm{Cr}(\mathrm{VI})$ removal from wastewater including iron nanoparticles [6], graphene oxide [7], carbon nanotubes [8], and metal-organic frameworks(MOFs) [9]. Ling used sodium borohydride synthesized iron nanoparticles to remove $\mathrm{Cr}(\mathrm{VI})(66 \%)$ [6]. Mondal synthesized graphene oxide using Hummer and Offeman's techniques for removing $\mathrm{Cr}(\mathrm{VI})$ under the $\mathrm{pH}$ of 4 , and documented a removal efficiency of $92.8 \%$ [7]. Ahmed et al., using the chemical vapor deposition method synthesized a high purity carbon nanotube to remove $\mathrm{Cr}(\mathrm{VI})$ with a sorption capacity of around $333.30 \mathrm{mg} \cdot \mathrm{g}^{-1}$ [8]. Zhang et al. prepared 2-cationic MOFs, FIR-53 and FIR-54 through the nanoscale route and this reported a removal capacity of $100 \mathrm{mg} \cdot \mathrm{g}^{-1}$ for $\mathrm{Cr}(\mathrm{VI})$ [9].

One type of nanomaterial commonly proposed for wastewater treatment is iron-based nanoparticles $(n \mathrm{Fe})$, because they possess the advantages of higher intrinsic reactivity on their surface sites due to their small particle size, large specific surface area and the presence of zero-valent iron which is often suitable for the reductive dechlorination of organic molecules [10]. Thus, $n \mathrm{Fe}$ is frequently used for the urgent removal of various organic and inorganic contaminants from surface or ground waters [11]. Existing traditional methods for $n$ Fe synthesis include chemical reduction, hydrothermal synthesis and physical vapor deposition, where chemical liquid-phase reduction is the most widely used method. However, synthesis via chemical reduction has a number of major issues including, firstly, toxicity to natural organisms, and the agglomeration of nanoscale materials leads to a greatly reduced effective contact area and deteriorating remediation performance [12]; secondly, the potential for nanoparticles to pose potential environmental hazards due to their higher solubility in water compared to micron-sized particles. Consequently, in an effort to reduce large-scale production costs and reduce the biological toxicity of traditionally produced $n \mathrm{Fe}$, alternative methods for the synthesis of environmentally friendly, low-cost, and reliable nano-iron materials have been the subject of much research attention in recent years [13].

During the green synthesis of $n \mathrm{Fe}$, simple iron salts are reduced to zero-valent iron by biomolecules contained in natural plant extracts $[13,14]$. Compared with traditional synthesis technologies, green synthesis avoids the use of innately toxic and hazardous chemicals, reduces energy consumption, and thus has the advantages of being environmentally friendly and more easily dispersed. Several natural plants, including green tea and eucalyptus leaves, have been used for the synthesis of iron-based nanoparticles [13,14], which have thereafter been shown to be ideal for the elimination of dyes, halogenated hydrocarbons and heavy metals [15-17]. The advantages of using plant extracts for green synthesized iron nanoparticles include simplicity, high efficiency, and sustainability. Recently, oolong tea extracts were used to synthesize predominantly spherical $n \mathrm{Fe}$ with diameters ranging from 40 to $50 \mathrm{~nm}$ for the degradation of malachite green (MG) dye, removing $75 \%$ of an initial MG dose of $50 \mathrm{mg} \cdot \mathrm{L}^{-1}$ with pseudo first-order reaction kinetics [15]. In addition, $n$ Fe synthesized using three different tea extracts served as a catalyst for the Fenton-like oxidation of monochlorobenzene (MCB), degrading $69 \%, 53 \%$, and $39 \%$ of MCB when using green, oolong, and black tea extracts, respectively. This study indicated that the type of extract had some effect on the resulting $n$ Fe degradation efficiency.

Green tea extract synthesized $n$ Fe demonstrated the best overall degradation, based on the initial adsorption of MCB to the $n$ Fe surface, with the decomposition of $\mathrm{H}_{2} \mathrm{O}_{2}$ resulting in hydroxyl radical generation which consequently resulted in MCB oxidation [12]. While application of zero-valent iron in metal remediation has been less commonly proposed, recently $n$ Fe synthesized using an eucalyptus leaf extract showed $100 \% \mathrm{Cr}(\mathrm{VI})$ removal efficiency at a leaf extract and iron (III) solution ratio of 2:1 (v/v) and $\mathrm{pH} 4$ [13]. In this study, the $n$ Fe produced had a diameter of $95 \mathrm{~nm}$ with FT-IR indicating a capping layer containing polyphenols and aliphatic acids [11]. Meanwhile, XPS revealed that $n$ Fe contained both iron oxides and a covering layer of eucalyptus leaf extract-derived biomolecules. However, despite these successes, some knowledge gaps in $n$ Fe synthesized by green tea extract still exist, including how specific synthetic conditions impact on the activity of $n \mathrm{Fe}$ in general and their efficiency for $\mathrm{Cr}(\mathrm{V})$ removal. To address these important discrepancies in our 
knowledge, this study specifically examined: (1) how the synthetic conditions affected the formation of $n \mathrm{Fe}$ and their $\mathrm{Cr}(\mathrm{VI})$ removal efficiency; (2) the specific biomolecules present in green tea extract and involved in the formation of $n \mathrm{Fe}$.

Therefore, the main objectives of this work were to: (1) investigate the optimal conditions for $n$ Fe synthesis; (2) characterize the $n$ Fe so formed; (3) analyze the main biomolecules in the green tea extract which act as reducing or capping agents; (4) propose a formation mechanism of $n$ Fe when using green tea extract.

\section{Materials and Methods}

\subsection{Materials}

Green tea was purchased from local tea factories in Hangzhou, Zhejiang Province. $\mathrm{FeSO}_{4} \cdot 7 \mathrm{H}_{2} \mathrm{O}$ was supplied by Sinopac Chemical Reagent Co. Ltd. (Danyang, Jiangsu, China). Commercial $n$ Fe was provided by Beijing Deke Daojin Science and Technology Co., Ltd. (Beijing, China). The sample was synthesized by sodium borohydride, and its specific surface area was $20 \mathrm{~m}^{2} \cdot \mathrm{g}^{-1}$ and average size amounted to $50 \mathrm{~nm}$. All chemical reagents were analytically pure.

\subsection{Synthesis of $n F e$}

Prior to the iron nanoparticle $(n \mathrm{Fe})$ being synthesized, the purchased green tea was washed liberally with distilled water and dried for $12 \mathrm{~h}$ at $333 \mathrm{~K}$. Thereafter, an aliquot of the dried green tea $(40 \mathrm{~g})$ and distilled water $(1 \mathrm{~L})$ was placed in a $1 \mathrm{~L}$ conical flask and heated in a water bath for $60 \mathrm{~min}$ at four different temperatures $(313,333,353$, and $373 \mathrm{~K})$, prior to cooling to room temperature and filtering through $0.45 \mu \mathrm{m}$ disposable filters to obtain a $40 \mathrm{~g} \cdot \mathrm{L}^{-1}$ green tea extract (GT). An iron slat solution was prepared by dissolving $\mathrm{FeSO}_{4} \cdot 7 \mathrm{H}_{2} \mathrm{O}(27.8 \mathrm{~g})$ in distilled water $(1 \mathrm{~L})$. Thereafter, aliquots of this solution were mixed with GT (30 mL) at five different volume ratios (1:3, 1:2, 1:1, 2:1, and 3:1) in a $150 \mathrm{~mL}$ conical flask, where the synthesized $n$ Fe was facilitated by heating it in a constant temperature oscillating chamber $(298,308,318$ or $318 \mathrm{~K}, 150 \mathrm{rpm})$ for $60 \mathrm{~min}$. Then, the $n \mathrm{Fe}$ sample was washed three times using anhydrous ethanol and deionized water, respectively.

\subsection{Characterization}

Surface micromorphology and structure of the green tea extract-derived $n \mathrm{Fe}$ was analyzed using Merlin Compact scanning electron microscopy (SEM) (Zeiss, Baden-Wurttemberg, Germany) and transmission electron microscopy (TEM) (Micrometer, JEOL, Beijing, China). To determine functional groups on the $n \mathrm{Fe}$ surface the analysis was done employing Fourier transform infrared spectra (FT-IR) (Nicolet IS 50, Thermo Fisher, Waltham, Massachusetts, USA) and the recording range was set at 400 to $4000 \mathrm{~cm}^{-1}$. Surface chemical compositions of $n$ Fe were analyzed using X-ray photoelectron spectroscopy (XPS) (AXIS Supra, Kratos Analytical, Trafford Park, Manchester, UK). Meanwhile the surface area and nanoparticle size were analyzed using $\mathrm{N}_{2}$-Brunauer-Emmett-Teller isotherm (BET) (ASAP 2020 Plus HD88, Shanghai, China).

The composition of the green tea extract before and after $n \mathrm{Fe}$ synthesis was analyzed using a gas chromatography-mass spectrometer (GC-MS) on a 7890B Agilent GC instrument equipped with a mass selective detector (MSD) (HP 5977C) quadrupole MS (Palo Alto, Agilent Technologies, Palo Alto, California, USA). Helium (99.999\%) at a flow rate of $1.0 \mathrm{~mL} \cdot \mathrm{min}^{-1}$ served as the carrier gas while chromatographic separation was conducted through a HP-5MS $(30 \mu \mathrm{m} \times 250 \mu \mathrm{m} \times 0.25 \mu \mathrm{m})$ capillary column. Samples were introduced into the GC-MS system in splitless mode and the injector and ion source temperatures were set to 280 and $230{ }^{\circ} \mathrm{C}$, respectively. The GC oven temperature was initially maintained at $50{ }^{\circ} \mathrm{C}$ for $2 \mathrm{~min}$, prior to being increased from 50 to $280{ }^{\circ} \mathrm{C}$ at a heating rate of $6^{\circ} \mathrm{C} \cdot \mathrm{min}^{-1}$. The GC-MS operating mode was set to use $70 \mathrm{eV}$ of ionization energy for electron bombardment, and the temperatures of the quadrupole and ionization source were set to 150 and $250{ }^{\circ} \mathrm{C}$, respectively. The mass spectra were obtained for the $\mathrm{m} / \mathrm{z}$ ratio in a full scan range with a 5.5 min solvent delay. 


\subsection{Removal of $\mathrm{Cr}(\mathrm{VI})$ by $n \mathrm{Fe}$}

The removal efficiency of $n \mathrm{Fe}$ was investigated using a batch process. This study used the $n \mathrm{Fe}$ synthesized under optimal conditions. These conditions included a GT concentration of $40 \mathrm{~g} \cdot \mathrm{L}^{-1}$, a GT:Fe ${ }^{2+}$ ratio of 1:2, a GT extract temperature of $353 \mathrm{~K}$ and a synthesis room temperature of $298 \mathrm{~K}$. During batch studies, a specific dose of $n \mathrm{Fe}$ was exposed to an $80 \mathrm{mg} \cdot \mathrm{L}^{-1}$ solution of $\mathrm{Cr}(\mathrm{VI})(50 \mathrm{~mL})$ for predefined times $(2,4,6,8,10$, and $12 \mathrm{~h}$ ) at $298 \mathrm{~K}$ and $150 \mathrm{rpm}$ in an incubator. At each sampling time, an aliquot of the supernatant reaction solution was removed and filtered $<0.45 \mu \mathrm{m}$ to measure the absorbance of $\mathrm{Cr}(\mathrm{VI})$ at $540 \mathrm{~nm}$ using an ultraviolet and visible spectrophotometer (UV-vis) (UV3600PLUS, Shimadzu).

\section{Results and Discussion}

\subsection{Effect of Synthesizing Conditions on Removal Efficiency}

In order to obtain superior functional $n \mathrm{Fe}$, various synthesis conditions involving GT bath temperature, synthesis temperature and $\mathrm{Fe}^{2+}$ to green tea extract ratio of $n \mathrm{Fe}$ were controlled to make possible the removal of hexavalent chromium (Figure 1). The absorbance of $\mathrm{Cr}(\mathrm{VI})$ was measured using an UV-vis in this paper, which mainly focused on the removal of $\mathrm{Cr}(\mathrm{VI})$.
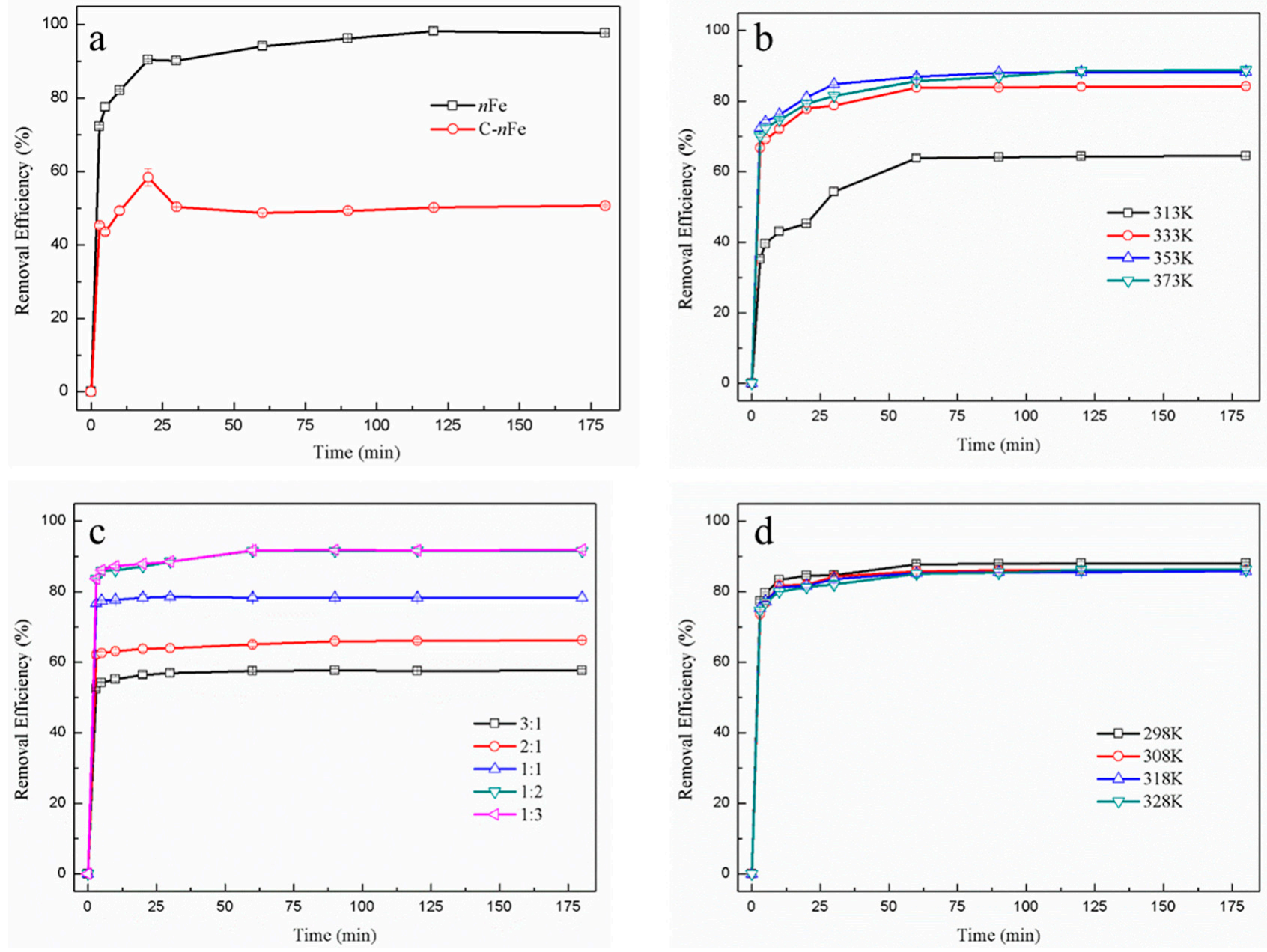

Figure 1. Temporal variation in $\mathrm{Cr}(\mathrm{VI})$ removal efficiency for green synthesized $n$ Fe (black line) and commercially purchased $n \mathrm{Fe}(\mathrm{C}-n \mathrm{Fe})$ (red line) (a) with different synthesis conditions, including green tea extract water bath temperature (313, 333, 353 , and $373 \mathrm{~K})(\mathbf{b})$; the ratio of GT:Fe ${ }^{2+}(3: 1,2: 1,1: 1,1: 2$, and 1:3) (c); synthesis temperature $(298,308,318$, and $328 \mathrm{~K})(\mathbf{d})$. 
The removal efficiency of $\mathrm{Cr}(\mathrm{VI})$ using green synthesized $n \mathrm{Fe}$ produced here $(97.66 \%)$ was much higher than that of commercially sourced $n \mathrm{Fe}(50.68 \%)$ at $180 \mathrm{~min}$ (Figure 1a). This improvement in $\mathrm{Cr}(\mathrm{VI})$ removal efficiency when utilizing green synthesized material might be attributed to the presence of biomolecules on the $n$ Fe surface, which contain a myriad of carboxyl, hydroxyl and other functional groups. They can combine $\mathrm{Cr}(\mathrm{VI})$ to form a covalent bond, resulting in much better removal efficiency. On the other hand, biomolecules on the $n$ Fe surface can reduce the agglomeration of $n$ Fe to enhance efficiency in $\mathrm{Cr}(\mathrm{VI})$ removal. Irrespective of the final $n \mathrm{Fe}$ sources, the time to reach equilibrium for both was about $30 \mathrm{~min}$, suggesting that the iron particles synthesized by green tea had the same overall function as that of the commercially available sample. Nonetheless they proved to be more efficient in removal capacity.

While the $\mathrm{Cr}(\mathrm{VI})$ removal efficiency tended to increase when the green tea extract temperature also rose (Figure $1 \mathrm{~b}$ ) and reached its highest $(88.25 \%$ ) at $353 \mathrm{~K}$, the removal efficiency was not significantly higher for the three temperatures above $313 \mathrm{~K}(333,353$, and $373 \mathrm{~K})$. This suggested that some biomolecules in green tea important for $\mathrm{Cr}(\mathrm{VI})$ removal were not efficiently extracted when the water batch temperature was $<333 \mathrm{~K}$, potentially resulting in less functionalized $n \mathrm{Fe}[18,19]$.

The ratio of GT:Fe ${ }^{2+}$ used in the synthesis of $n$ Fe affected the $\mathrm{Cr}(\mathrm{VI})$ removal efficiency (Figure 1c). For ratios of GT:Fe ${ }^{2+}$ as low as 1:2, the removal efficiency remained unchanged. However, as the GT: $\mathrm{Fe}^{2+}$ ratio increased from 1:2 to 1:3, there was a subsequently slight increase in the removal efficiency from $91.6 \%$ and $91.9 \%$, respectively. These results generally showed that $\mathrm{Cr}(\mathrm{VI})$ removal efficiency increased with $\mathrm{Fe}^{2+}$, indicating that the amount of Fe in the $n$ Fe played an important role in $\mathrm{Cr}(\mathrm{VI})$ removal $[20,21]$. Besides, the overall difference in removal efficiency was minor, because the biological molecular weight of green tea extract may not be enough to synthesize iron nanoparticles from excess iron divalent ions [22].

The removal efficiency of $\mathrm{Cr}(\mathrm{VI})$ did not significantly alter when synthesis temperature varied between 298 and $328 \mathrm{~K}$ (Figure 1d). Consequently, temperature did not affect $n \mathrm{Fe}$ activity. Considering the large-scale economics of production, the best scenario for $n \mathrm{Fe}$ synthesis would be simple room temperature.

During synthesis, the best $\mathrm{Cr}(\mathrm{VI})$ removal was obtained at a GT:Fe${ }^{2+}$ ratio of $1: 2$, at a temperature to leach GT of $353 \mathrm{~K}$ and a temperature to synthesize $n \mathrm{Fe}$ at room temperature. The removal efficiency of green synthesized $n \mathrm{Fe}$ for hexavalent chromium was much higher than that of commercially purchased $n \mathrm{Fe}$, which suggests that green tea molecules may wrap around the $n \mathrm{Fe}$, while the functional groups of green tea molecules could form chemical bonds with hexavalent chromium for removal. Whether $\mathrm{Cr}(\mathrm{VI})$ could be reduced to $\mathrm{Cr}$ (III) by $n \mathrm{Fe}$ and remain in solution was not clear. Atomic emission spectroscopy can detect the valence and concentration of both $\mathrm{Cr}(\mathrm{VI})$ and $\mathrm{Cr}(\mathrm{III})$, which has been documented in one study [23]. The structure and morphology of $n$ Fe prepared under these optimal conditions were subsequently examined to understand the process.

\subsection{Nanoparticle Characterization}

Consistent with previous studies [24,25], SEM revealed that the produced $n$ Fe were uniformly dispersed, spherical in shape and with an average diameter of 50-80 nm (Figure 2). A few agglomerated particles were more than $100 \mathrm{~nm}$ in size with a chain-like structure, mainly due to the strong magnetism and van der Waals attraction between $n \mathrm{Fe}$ [26,27]. It was thus concluded that the use of GT during $n$ Fe synthesis is beneficial, since GT acted as a dispersant during synthesis and reduced agglomeration. In order to further understand the internal structure and morphology of $n \mathrm{Fe}$, TEM characterization was performed below (Figure 3). 


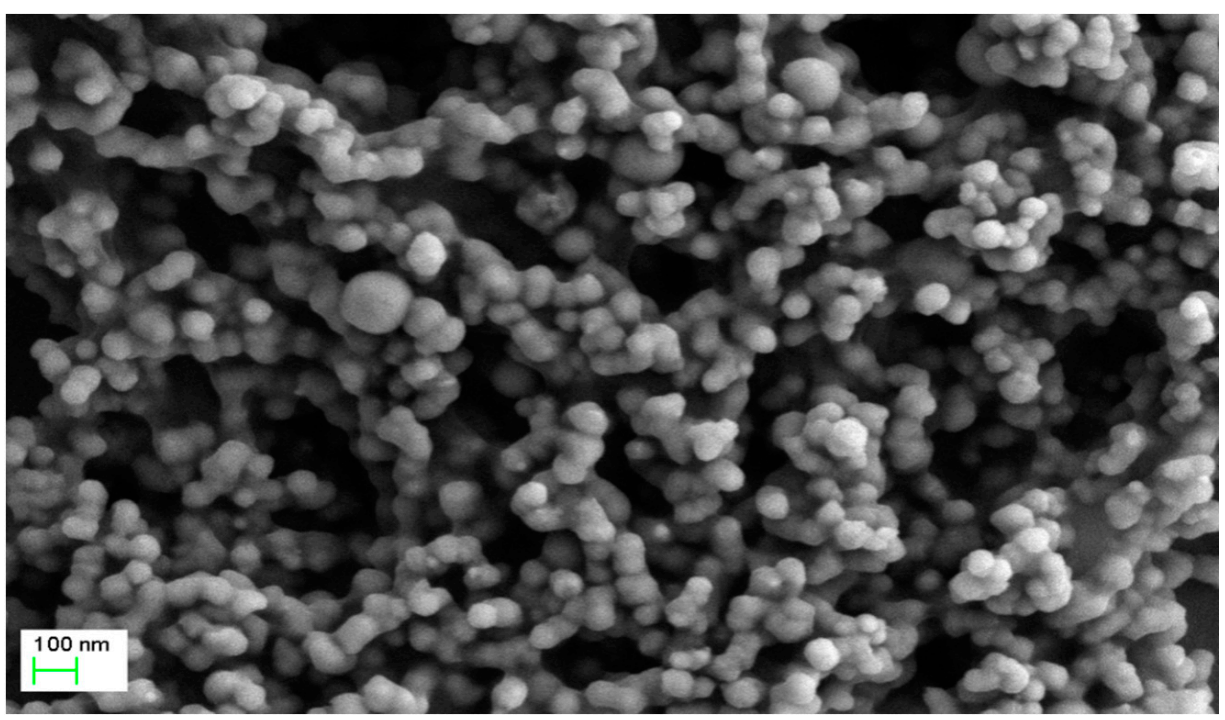

Figure 2. SEM image of $n$ Fe prepared using green tea extract. Conditions: accelerating voltage of $3 \mathrm{kV}$ and indicated magnification of $20 \mathrm{k} \times$.

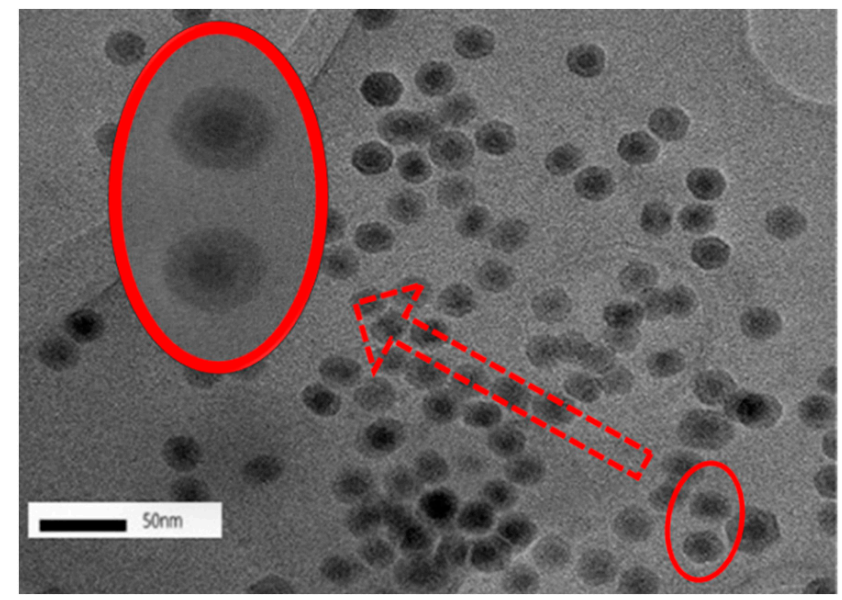

Figure 3. TEM images of $n$ Fe. Conditions: accelerating voltage of $200 \mathrm{kV}$, indicated magnification of $100 \mathrm{k} \times$.

Under magnification, the TEM images indicated that the $n$ Fe exhibited a core-shell structure (Figure 3). The core-shell structure has been reported previously [28], where zero-valent iron nanoparticles constitute a core surrounded by a layer of oxidized iron. It has been reported that the $n$ Fe was well dispersed with little agglomeration, which was attributed to the organic compounds derived from the bioactive substances in the green tea extract. This acted as a protective dispersion and stabilizer, reducing both oxidization and agglomeration [17]. Furthermore, the dynamic light scattering (DLS) helped to confirm "non clustering" according to one report [14]. While the observance of a core-shell structure is fairly common, using green tea extract to coat the formed $n$ Fe with biomolecules might contribute to some differences from the chemically synthesized $n$ Fe [29,30]. To further understand the valence state of the central iron nanoparticles and the nature of the GT coating, XPS characterization was carried out.

The full range XPS scan image of the fabricated nanoparticles indicated they contained three main elements, Fe, C, and O (Figure 4A), confirming that biomolecules derived from the green tea extract were located on the iron nanoparticles' surfaces [17]. This was consistent with the TEM analysis. Agreeing with previous studies [31,32], peak fitting of the iron region (Figure 4B) indicated that the valence of the iron was distributed between Fe (II) and Fe (0). This further confirmed that zero-valent iron nanoparticles were likely to 
be present in the core, proving that the green tea extract had reduced $\mathrm{Fe}^{2+}$ to $\mathrm{Fe}(0)$ and thus acted as a reducing agent. It was, however, surrounded by a layer of ferrous oxide nanoparticles forming a core-shell structure. In order to determine the adsorption behavior and specific surface area of $n \mathrm{Fe}$, BET characterization was carried out as follows.
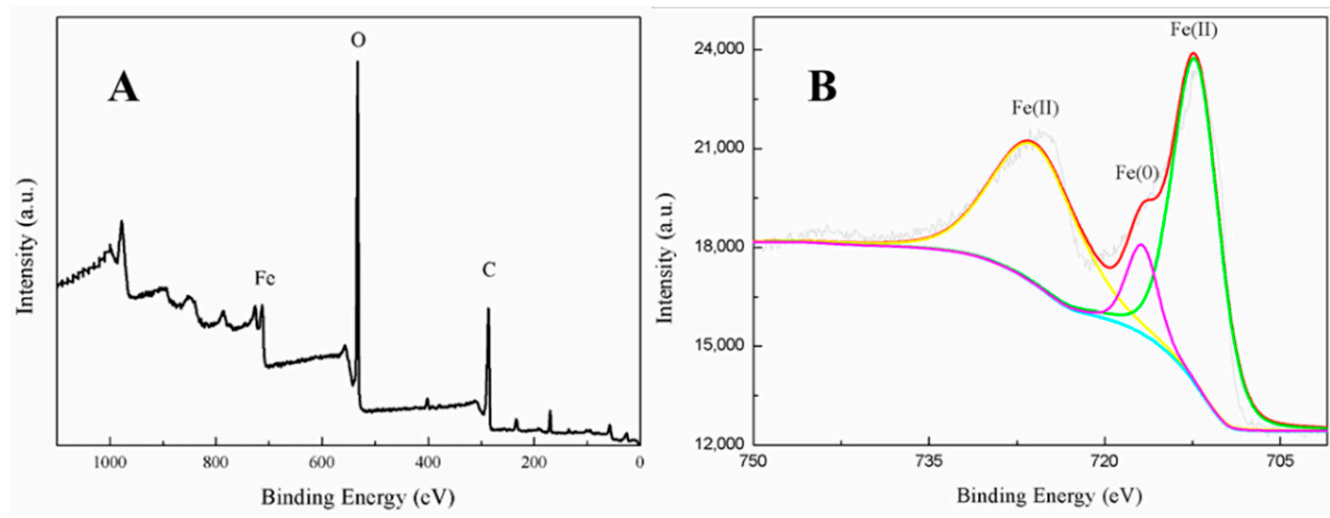

Figure 4. XPS images of $n \mathrm{Fe}$, full scan image (A) and expanded Fe region (B).

The $\mathrm{N}_{2}$ Brunauer-Emmett-Teller isotherm (BET) of $n$ Fe (Figure 5) exhibited a type IV nitrogen adsorption-desorption isotherm character, indicative of multi-layer adsorption. The adsorption-desorption hysteresis loop was classified as $\mathrm{H} 3$, suggesting that the $n \mathrm{Fe}$ might contain slit-like pores [33]. The specific surface area of $n \mathrm{Fe}$ was $42.25 \mathrm{~m}^{2} \cdot \mathrm{g}^{-1}$, which was larger than that of commercially purchased $n \mathrm{Fe}\left(20 \mathrm{~m}^{2} \cdot \mathrm{g}^{-1}\right)$, meaning that the overall $n \mathrm{Fe}$ synthesized by GT had a large specific surface area. This was attributed to the organic compounds derived from the bioactive substances in the GT acting as a protective dispersion and stabilizer. It could therefore be concluded that SEM, TEM and the large specific area probably contributed to the good efficiency in removing $\mathrm{Cr}(\mathrm{VI})$ [17].

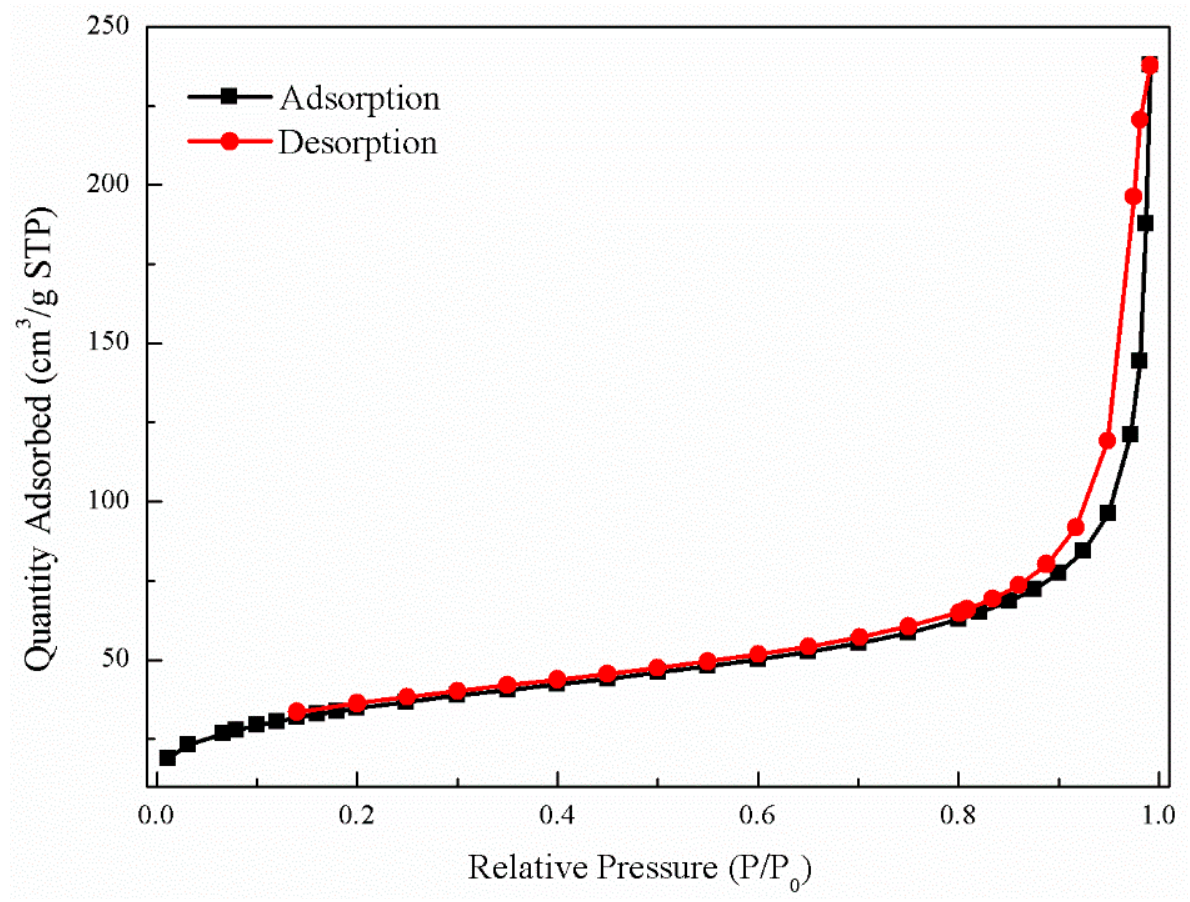

Figure 5. BET isotherm of $n \mathrm{Fe}$.

\subsection{Analysis of the Green Tea Extract}

In order to further determine the contribution of green tea to the core-shell structure of the iron nanoparticles, FT-IR and GC-MS characterizations were reported in this section. 
Comparison of the FT-IR spectra of GT and $n$ Fe (Figure 6) showed that the peak at $1611.7 \mathrm{~cm}^{-1}$ initially present in the green tea extract disappeared after the $n \mathrm{Fe}$ was synthesized. This peak was attributed to $C=C$ stretching vibrations [34], and its disappearance indicated that biomolecules containing $\mathrm{C}=\mathrm{C}$ bonds were involved in the synthesis of $n \mathrm{Fe}$. Peaks at 1362 and $1039 \mathrm{~cm}^{-1}$ in the GT extract, attributable to C-N and C-O-C, respectively, remained largely unchanged following synthesis. It suggests that biomolecules in green tea played a role in the reductive synthesis of $n \mathrm{Fe}$, as well as capping agents. Additionally, in the fabricated $n \mathrm{Fe}$ a new peak appeared at $869.2 \mathrm{~cm}^{-1}$, one that was not present in the green tea extract which was ascribed to Fe-O stretching in $\mathrm{FeOOH}$ [35]. This scenario is consistent with that of XPS and confirmed that the nanometer iron oxide in the shell was FeOOH. Overall, the FT-IR results were consistent with those from XPS and further supported the presence of iron oxides, and that, while biomolecules containing $\mathrm{C}=\mathrm{C}$ played a role in the iron reduction, other biomolecules containing $\mathrm{C}-\mathrm{N}$ and $\mathrm{C}-\mathrm{O}-\mathrm{C}$ acted more as capping agents. Subsequently, GC-MS analysis of the extracts before and after synthesis were carried out to specifically identify which biomolecules were likely to be involved.

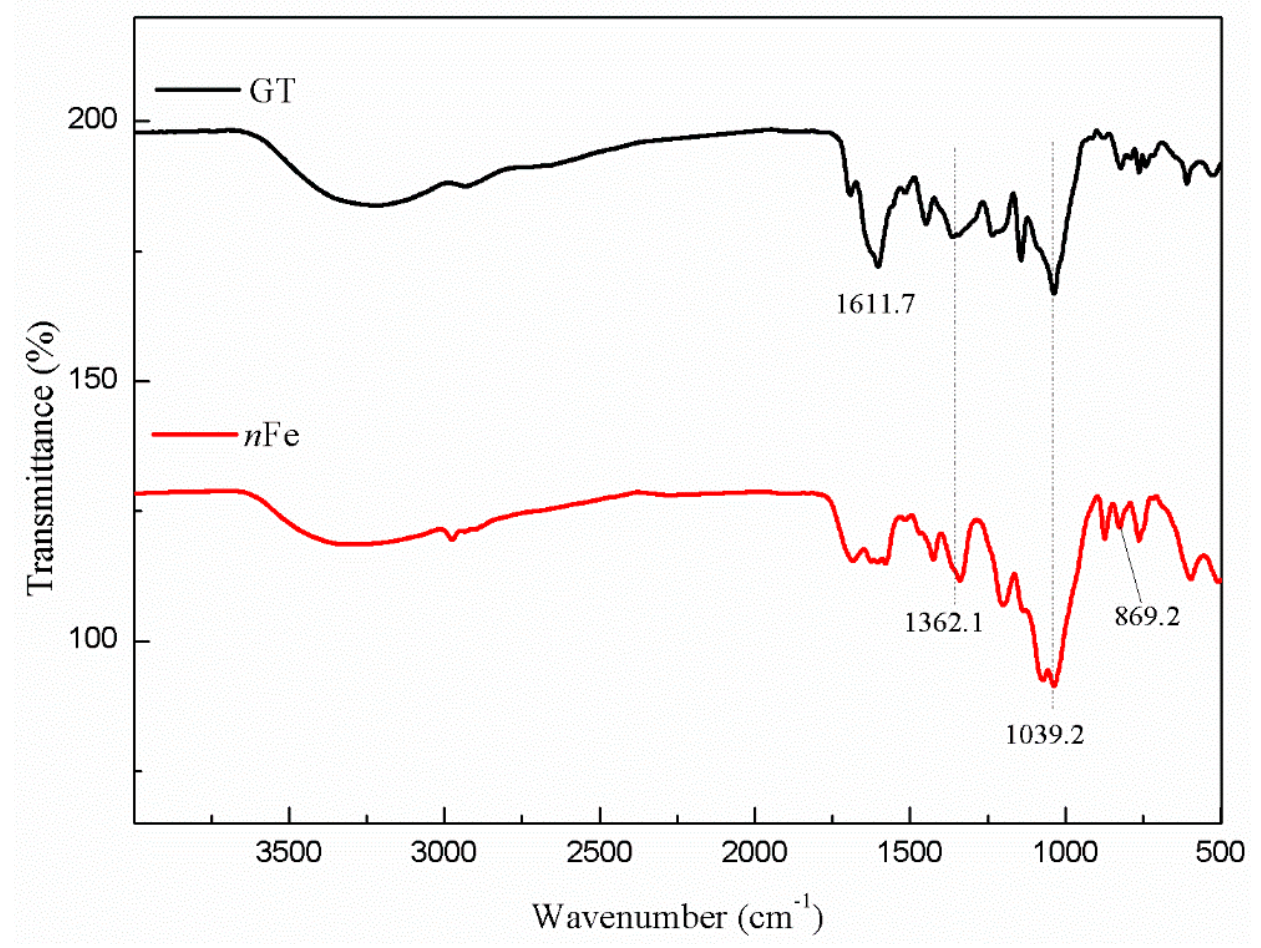

Figure 6. FT-IR spectra of GT (black line) and $n$ Fe (red line).

Comparison of the GT extract before and after the $n$ Fe synthesis (Figure 7) confirmed that of the eight peaks present in the original GT extract, seven significant peaks completely disappeared (1, 2, 3, 4, 5, 7, and 8) and one peak significantly decreased (6) following $n$ Fe synthesis. This indicated that all of these biomolecules were significantly involved in $n \mathrm{Fe}$ synthesis. Moreover, since no new peaks appeared after synthesizing $n \mathrm{Fe}$, this suggested that the biomolecules originally present were not transformed during synthesis. The specific biomolecules present were identified from the examination of their mass fragmentation pattern and comparison to the MS library match (Table 1). The main constituents of the GT were therefore phenol (11.47 min), 1,1'-Biphenyl, 2-ethyl (16.37 $\mathrm{min})$, 1,2,3-Benzenetriol (17.71 $\mathrm{min})$, 1,3,5-Benzenetriol (24.70 $\mathrm{min}$ ), 6-Hydroxy-4,4,7a-trimethyl5,6,7a-tetrahydrobenzofuran (25.33 $\mathrm{min})$, caffeine (27.14 $\mathrm{min})$, oleanitrile (36.09 $\mathrm{min})$, and bis (2-ethylhexyl) phthalate (36.80 min). These results were consistent with those of FT-IR and several previous studies which demonstrated that such GT biomolecules acted both as reducing and capping agents [17]. 


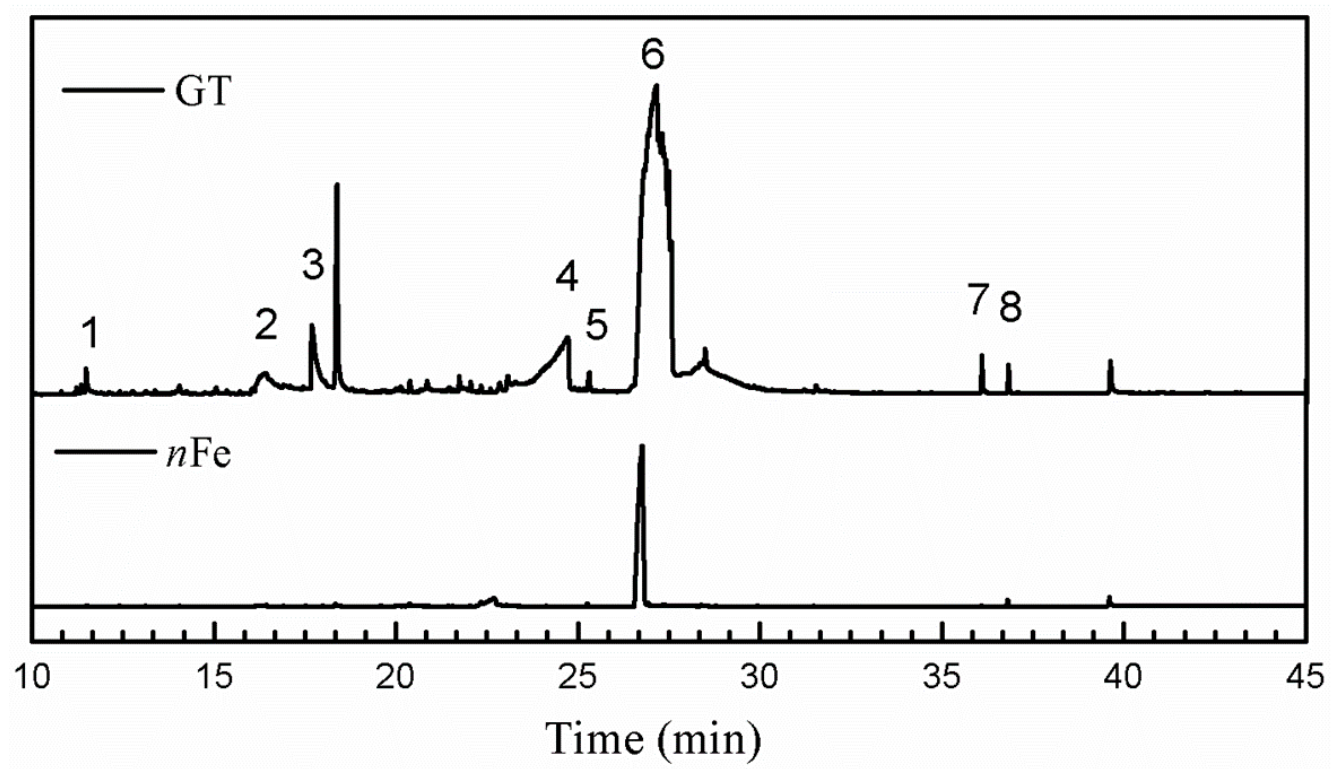

Figure 7. GC-MS chromatograms of GT (upper line) and $n$ Fe (lower line).

Table 1. Mass spectrometry classification of the main biomolecules in GT.

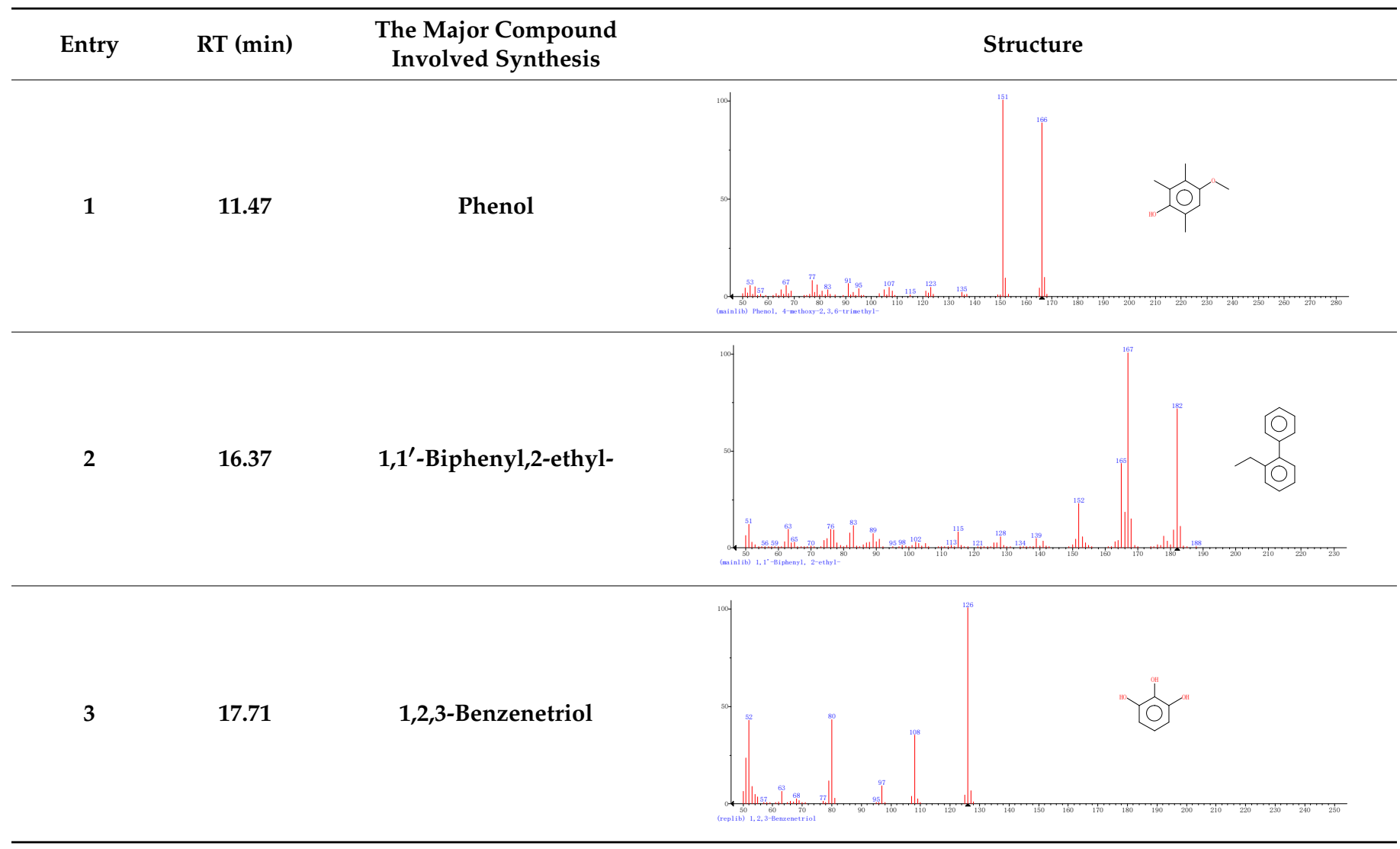


Table 1. Cont

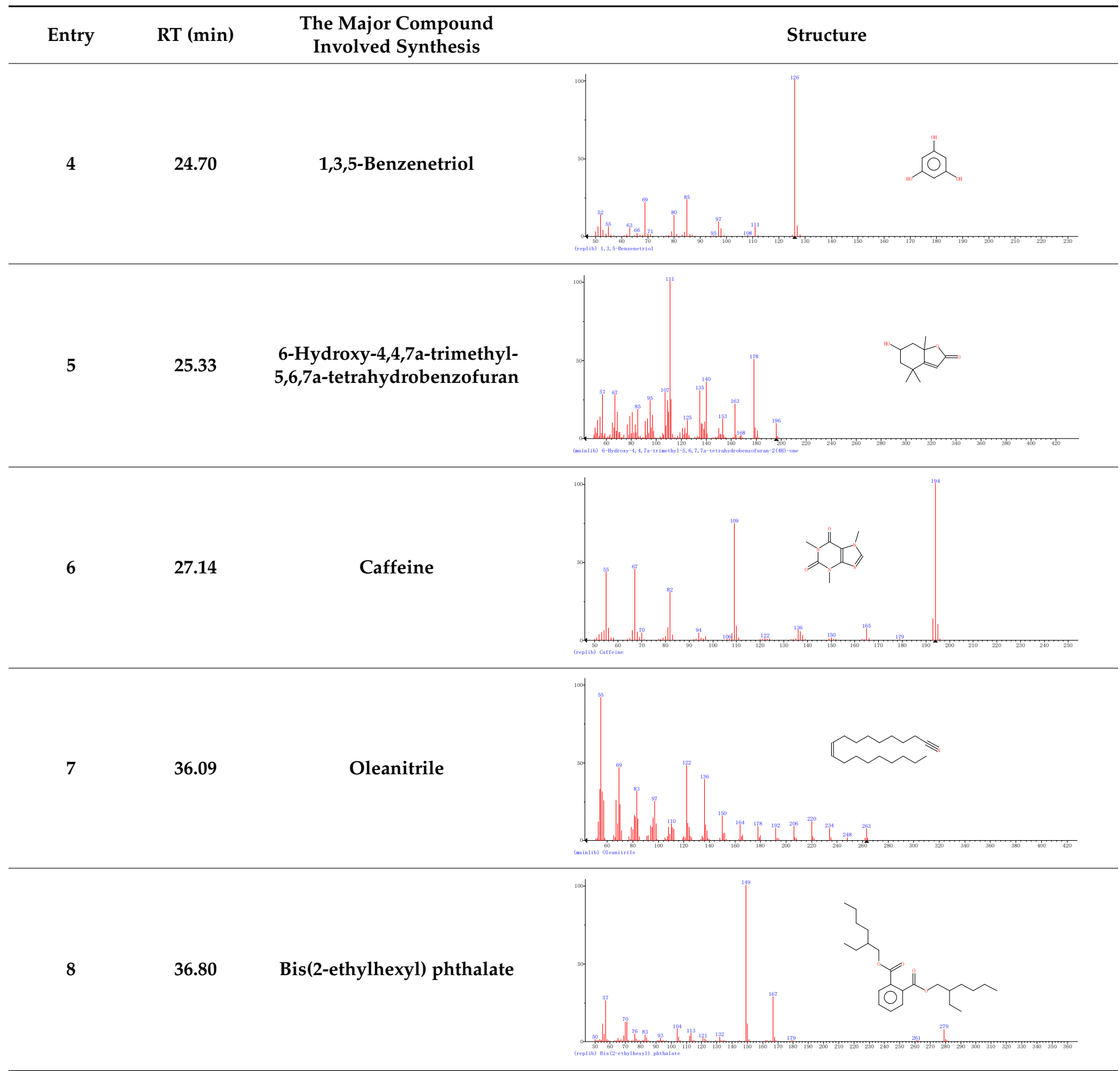

\subsection{Mechanism}

When considering all the results holistically, this makes it possible to propose a mechanism for synthesizing green tea extract-derived $n$ Fe (Figure 8). Initially, biomolecules present in green tea, such as 1,3,5-Benzenetriol, 1,2,3-Benzenetriol, caffeine and bis(2-ethylhexyl) phthalate, were extracted into an aqueous solution, which, upon mixing with an iron salt, spontaneously led to the formation of core-shell structured $n \mathrm{Fe}$. It comprised an $\mathrm{Fe}(0)$ core and FeOOH shell, where all of the major biomolecules in the green tea extract acted as the reducing and capping agents, functioning to reduce $\mathrm{Fe}^{2+}$ to $\mathrm{Fe}(0)$ and cap around the iron nanoparticles. 


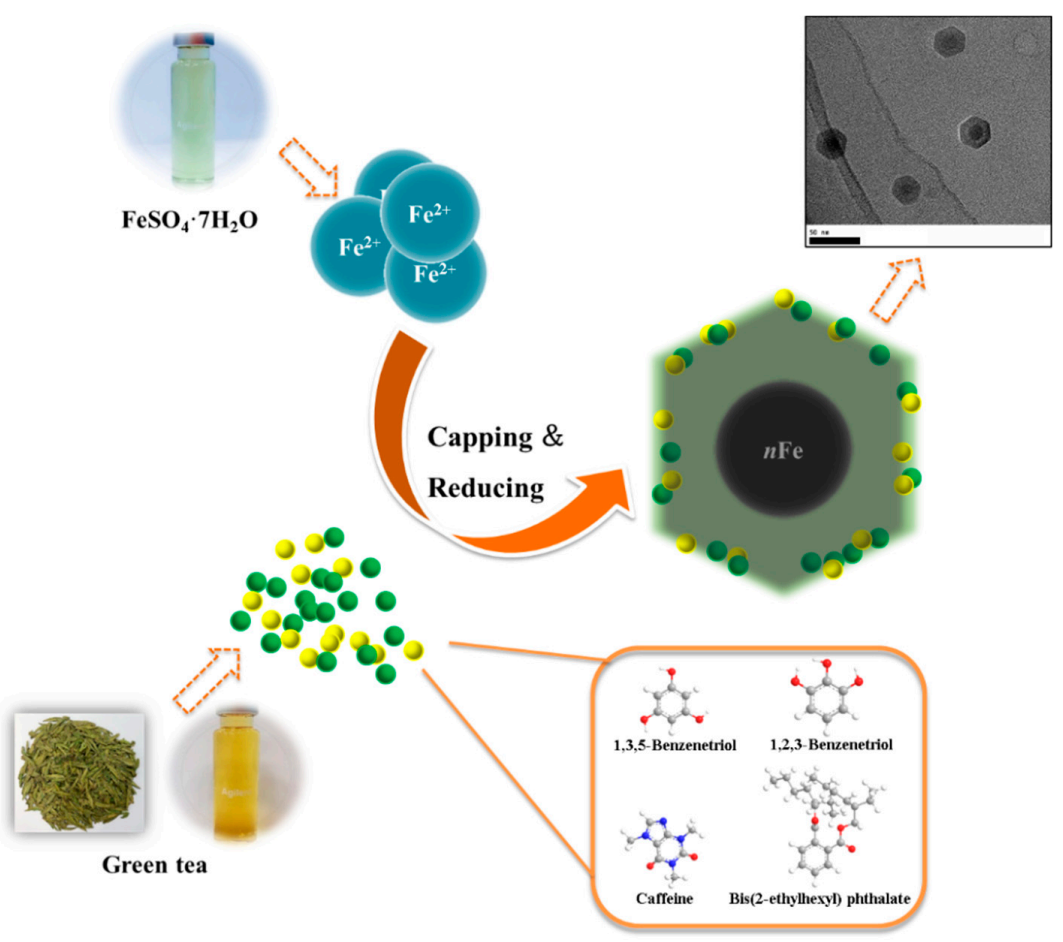

Figure 8. Proposed mechanism of the green tea synthesis of $n$ Fe.

\subsection{Stability of $n F e$ Removal Efficiency}

The long-term stability of the produced nanoparticles in terms of the sustained removal efficiency when exposed to air is an important consideration for long-term practical water treatment. In many cases $n$ Fe activity can be significantly reduced due to passivation via iron-oxide surface formation when exposed to air. Here, the $\mathrm{Cr}(\mathrm{VI})$ removal efficiency when continually exposed to air remained stable and high (around 85\%) for up to $12 \mathrm{~h}$ (Figure 9). This indicated that the material retained high stability, which was attributed to the surface coating of bioorganic molecules derived from the green tea extract. They completely encapsulated the iron nanoparticles and prevented oxidation and consequential inactivation. This outcome highlighted that green synthesized $n$ Fe has significant practical potential for water treatment strategies.

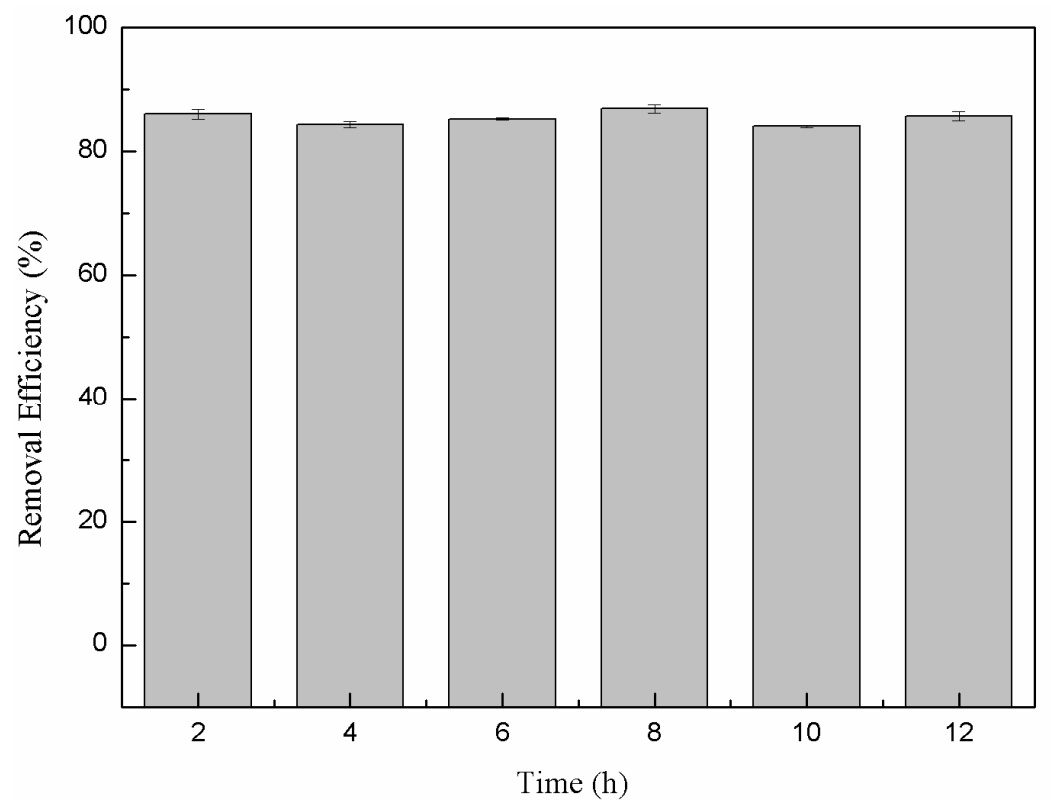

Figure 9. Variation in the removal efficiency of $n$ Fe over time. 


\section{Conclusions}

A green tea extract was successfully used for the highly efficient (91.6\%) green synthesis of $n \mathrm{Fe}$ to remove $\mathrm{Cr}(\mathrm{VI})$ from aqueous solutions. Control of synthesis conditions made it possible to optimize the functional removal efficiency of the nanomaterial produced. SEM and TEM confirmed that the material was a spherical particle with a core-shell structure. XPS showed that the zero-valent iron nanoparticles acted as the core, while iron oxide nanoparticles formed the shell; FT-IR confirmed that the iron oxide shell was $\mathrm{FeOOH}$. GC-MS suggested that most of the biomolecules present in the green tea extract, such as 1,2,3-Benzenetriol, 1,3,5-Benzenetriol, caffeine and bis(2-ethylhexyl) phthalate, were actively involved in the $n \mathrm{Fe}$ synthesis as reducing or capping agents, which provided a theoretical basis for the green synthesis of nanomaterials. These biomolecules reduced $\mathrm{Fe}^{2+}$ to $\mathrm{Fe}(0)$ and capped the $n \mathrm{Fe}$ surface to prevent oxidation and inactivation. This capping led to a high atmospheric stability so that the material could be practically applied more easily than existing materials for water treatment. However, the exact mechanism for removing $\mathrm{Cr}(\mathrm{VI})$ by green synthesized $n \mathrm{Fe}$ is still unclear. Whether green tea also plays a role in this process needs to be analyzed and validated in our next study.

Author Contributions: D.L., J.Z. and T.J. conceived and designed the experiments; R.H. and T.J. performed the experiments; D.L., R.H., and T.J. analyzed the data; D.L., T.J., and R.H. contributed reagents/materials/analysis tools; R.H. and T.J. wrote the paper. All authors have read and agreed to the published version of the manuscript.

Funding: This research was sponsored by a National Ministry of Environmental Protection Standard Preparation Project 2016-54, run by Beijing Environmental Protection Research Institute of Light Industry.

Acknowledgments: The authors would like to sincerely thank Gary Owens for his significant contributions in proofreading and editing this manuscript prior to submission. His valuable scientific insights, comments, and criticisms, including extensive reinterpretations and critical revisions, have certainly improved the quality of the manuscript.

Conflicts of Interest: The authors declare there is no conflict of interest.

\section{References}

1. Nur-E-Alam, M.; Mia, M.A.; Ahmad, F.; Rahman, M.M. An overview of chromium removal techniques from tannery effluent. Appl. Water Sci. 2020, 10, 205. [CrossRef]

2. Fathima, N.N.; Aravindhan, R.; Rao, J.R.; Nair, B.U. Solid Waste Removes Toxic Liquid Waste: Adsorption of Chromium(VI) by Iron Complexed Protein Waste. Environ. Sci. Technol. 2005, 39, 2804-2810. [CrossRef]

3. Almeida, J.C.; Cardoso, C.E.D.; Tavares, D.S.; Freitas, R.; Trindade, T.; Vale, C.; Pereira, E. Chromium removal from contaminated waters using nanomaterials-A review. Trend. Anal. Chem. 2019, 118, 277-291. [CrossRef]

4. Zhao, H.; Wang, R.; Deng, H.; Zhang, L.; Gao, L.; Zhang, L.; Jiao, T. Facile Preparation of Self-Assembled Chitosan-Based POSS-CNTs-CS Composite as Highly Efficient Dye Absorbent for Wastewater Treatment. ACS Omega 2021, 6, 294-300. [CrossRef] [PubMed]

5. Deng, H.; Yin, J.; Ma, J.; Zhou, J.; Zhang, L.; Gao, L.; Jiao, T. Exploring the enhanced catalytic performance on nitro dyes via a novel template of flake-network Ni-Ti LDH/GO in-situ deposited with $\mathrm{Ag}_{3} \mathrm{PO}_{4}$ NPs. Appl. Surf. Sci. 2021, 543, 148821. [CrossRef]

6. Ling, L.; Zhang, W.-X. Mapping the reactions of hexavalent chromium [Cr(vi)] in iron nanoparticles using spherical aberration corrected scanning transmission electron microscopy (Cs-STEM). Anal. Methods 2014, 6, 3211-3214. [CrossRef]

7. Mondal, N.K.; Chakraborty, S. Adsorption of Cr (VI) from aqueous solution on graphene oxide (GO) pre-pared from graphite: Equilibrium, kinetic and thermodynamic studies. Appl. Water Sci. 2020, 10, 1-10. [CrossRef]

8. Ahmed, A.; Abdulhakim, S.A.; Ishaq, K.; Saka, A.A.; Peter, M.S.; Chidinma, O.U. Carbon nanotubes: Mechanism, Langmuir Hinshelwood growth kinetics and it application for the removal of Chromium (VI). J. Membr. Sci. Technol. 2017, 7, 100174-100184.

9. Zhang, Q.; Yu, J.; Cai, J.; Zhang, L.; Cui, Y.; Yang, Y.; Chen, B.; Qian, G. A porous Zr-cluster-based cationic metal-organic framework for highly efficient Cr2O72- removal from water. Chem. Commun. 2015, 51, 14732-14734. [CrossRef]

10. Blundell, S.P.; Owens, G. Evaluation of enhancement techniques for the dechlorination of DDT by nanoscale zero-valent iron. Chemosphere 2021, 264, 128324. [CrossRef]

11. Zha, S.; Cheng, Y.; Gao, Y.; Chen, Z.; Megharaj, M.; Naidu, R. Nanoscale zero-valent iron as a catalyst for heterogeneous Fenton oxidation of amoxicillin. Chem. Eng. J. 2014, 255, 141-148. [CrossRef]

12. Li, R.; Jin, X.; Megharaj, M.; Naidu, R.; Chen, Z. Heterogeneous Fenton oxidation of 2,4-dichlorophenol using iron-based nanoparticles and persulfate system. Chem. Eng. J. 2015, 264, 587-594. [CrossRef] 
13. Weng, X.; Guo, M.; Luo, F.; Chen, Z. One-step green synthesis of bimetallic Fe/Ni nanoparticles by eucalyptus leaf extract: Biomolecules identification, characterization and catalytic activity. Chem. Eng. J. 2017, 308, 904-911. [CrossRef]

14. Jin, X.; Liu, Y.; Tan, J.; Owens, G.; Chen, Z. Removal of $\mathrm{Cr}(\mathrm{VI})$ from aqueous solutions via reduction and absorption by green synthesized iron nanoparticles. J. Clean. Prod. 2018, 176, 929-936. [CrossRef]

15. Huang, L.; Weng, X.; Chen, Z.; Megharaj, M.; Naidu, R. Synthesis of iron-based nanoparticles using oolong tea extract for the degradation of malachite green. Spectrochim. Acta Part A Mol. Biomol. Spectrosc. 2014, 117, 801-804. [CrossRef]

16. Kuang, Y.; Wang, Q.P.; Chen, Z.L.; Megharaj, M.; Naidu, R. Heterogeneous Fenton-like oxidation of mon-ochlorobenzene using green synthesis of iron nanoparticles. J. Colloid Interf. Sci. 2013, 410, 67-73. [CrossRef]

17. Liu, Y.; Jin, X.; Chen, Z. The formation of iron nanoparticles by Eucalyptus leaf extract and used to remove Cr(VI). Sci. Total. Environ. 2018, 627, 470-479. [CrossRef]

18. Wang, T.; Jin, X.; Chen, Z.; Megharaj, M.; Naidu, R. Green synthesis of Fe nanoparticles using eucalyptus leaf extracts for treatment of eutrophic wastewater. Sci. Total. Environ. 2014, 466-467, 210-213. [CrossRef]

19. Cai, X.; Gao, Y.; Sun, Q.; Chen, Z.; Megharaj, M.; Naidu, R. Removal of co-contaminants Cu (II) and ni-trate from aqueous solution using kaolin-Fe/Ni nanoparticles. Chem. Eng. J. 2014, 244, 19-26. [CrossRef]

20. Afzal, M.; Safer, A.M.; Menon, M. Green tea polyphenols and their potential role in health and disease. Inflammopharmacology 2015, 23, 151-161. [CrossRef]

21. Xu, Q.; Li, W.; Weng, X.; Owens, G.; Chen, Z. Mechanism and impact of synthesis conditions on the one-step green synthesis of hybrid RGO@ Fe/Pd nanoparticles. Sci. Total Environ. 2020, 710, 136308. [CrossRef]

22. Akhtar, M.S.; Panwar, J.; Yun, Y.-S. Biogenic Synthesis of Metallic Nanoparticles by Plant Extracts. ACS Sustain. Chem. Eng. 2013, 1, 591-602. [CrossRef]

23. Gheju, M. Hexavalent Chromium Reduction with Zero-Valent Iron (ZVI) in Aquatic Systems. Water Air Soil Pollut. 2011, 222, 103-148. [CrossRef]

24. Njagi, E.C.; Huang, H.; Stafford, L.; Genuino, H.; Galindo, H.M.; Collins, J.B.; Hoag, G.E.; Suib, S.L. Bio-synthesis of Iron and Silver Nanoparticles at Room Temperature Using Aqueous Sorghum Bran Extracts. Langmuir 2011, 27, 264-271. [CrossRef]

25. Shahwan, T.; Abu Sirriah, S.; Nairat, M.; Boyacı, E.; Eroğlu, A.; Scott, T.; Hallam, K. Green synthesis of iron nanoparticles and their application as a Fenton-like catalyst for the degradation of aqueous cationic and anionic dyes. Chem. Eng. J. 2011, 172, 258-266. [CrossRef]

26. Petcharoen, K.; Sirivat, A. Synthesis and characterization of magnetite nanoparticles via the chemical co-precipitation method. Mater. Sci. Eng. B 2012, 177, 421-427. [CrossRef]

27. Shi, L.-N.; Zhang, X.; Chen, Z.-L. Removal of Chromium (VI) from wastewater using bentonite-supported nanoscale zero-valent iron. Water Res. 2011, 45, 886-892. [CrossRef]

28. Ling, L.; Zhang, W.-X. Enrichment and Encapsulation of Uranium with Iron Nanoparticle. J. Am. Chem. Soc. 2015, 137, 2788-2791. [CrossRef] [PubMed]

29. Cho, J.S.; Lee, J.C.; Rhee, S.H. Effect of precursor concentration and spray pyrolysis temperature upon hdroxyapatite particle size and density. J. Biomed. Mater. Res. B Appl. Biomater. 2016, 104, 422-430. [CrossRef]

30. Rajendran, K.; Sen, S. Optimization of process parameters for the rapid biosynthesis of hematite nano-particles. J. Photochem. Photobiol. B Biol. 2016, 159, 82-87. [CrossRef] [PubMed]

31. Grosvenor, A.P.; Kobe, B.A.; Biesinger, M.C.; McIntyre, N.S. Investigation of multiplet splitting of Fe 2p XPS spectra and bonding in iron compounds. Surf. Interface Anal. 2004, 36, 1564-1574. [CrossRef]

32. Liu, L.; Li, W.; Xiong, Z.; Xia, D.; Yang, C.; Wang, W.; Sun, Y. Synergistic effect of iron and copper oxides on the formation of persistent chlorinated aromatics in iron ore sintering based on in situ XPS analysis. J. Hazard. Mater. 2019, 366, 202-209. [CrossRef] [PubMed]

33. Choy, J.H.; Yoon, J.B.; Jung, H.; Park, J.H. Structural evolution of $\mathrm{SiO}_{2}-\mathrm{ZrO}_{2}$ nano-sol intercalated clays upon pillaring reaction. J. Mater. Chem. 2003, 13, 557-562. [CrossRef]

34. Soni, R.; Shukla, D.P. Synthesis of fly ash based zeolite-reduced graphene oxide composite and its evalua-tion as an adsorbent for arsenic removal. Chemosphere 2019, 219, 504-509. [CrossRef]

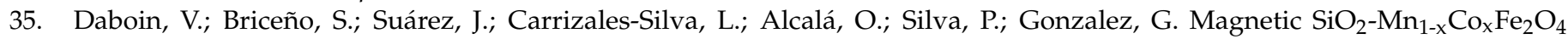
nanocomposites decorated with $\mathrm{Au} @ \mathrm{Fe}_{3} \mathrm{O}_{4}$ nanoparticles for hyperthermia. J. Magn. Magn. Mater. 2019, 479, 91-98. [CrossRef] 